\title{
Adjunctive Effects of Cyclosporine and Macrolide in Rapidly Progressive Cryptogenic Organizing Pneumonia with no Prompt Response to Steroid
}

\author{
Jaehee Lee ${ }^{1}$, Seung Ick Cha ${ }^{1}$, Tae In Park ${ }^{2}$, Jae Yong Park ${ }^{1}$, \\ Tae Hoon Jung ${ }^{1}$ and Chang Ho Kim ${ }^{1}$
}

\begin{abstract}
Cryptogenic organizing pneumonia (COP) generally responds well to corticosteroids with a favorable outcome. However, it can rapidly worsen and lead to respiratory failure that is refractory to corticosteroids. Adjunctive drugs have been used in refractory cases with various outcomes, but treatment experience is still lacking. We present a case of rapidly progressive COP accompanying air leak syndrome, which showed no prompt response to corticosteroids alone but gradual improvement with the addition of cyclosporine and macrolide. This case report supports the existing literature suggesting that an early therapeutic trial of this drug combination might be considered in COP patients whose condition worsens despite corticosteroid administration.
\end{abstract}

Key words: cryptogenic organizing pneumonia, air leak syndrome, cyclosporine, macrolide

(Intern Med 50: 475-479, 2011)

(DOI: 10.2169/internalmedicine.50.4237)

\section{Introduction}

Organizing pneumonia (OP) is a characteristic clinicopathologic syndrome that, in general, responds dramatically to steroids with a good outcome (1-4). However, some patients exhibit rapidly progressive disease that is often refractory to steroids and is associated with a poor outcome $(1,5-8)$. To date, anecdotes of successful cases in which non-steroidal agents have been used to treat patients with steroid-resistant OP have been rarely reported $(9,10)$. Thus, little information is currently available to aid in treating this condition. Herein, we introduce a patient with rapidly progressive cryptogenic organizing pneumonia (COP) complicated by air leak syndrome who responded well to the adjunctive use of cyclosporin A (CsA) and macrolide in combination with corticosteroid.

\section{Case Report}

A 38-year-old woman presented with a 2-week history of a worsening cough followed by progressive dyspnea. She had not been exposed to known toxins or dirty dust. Physical examination revealed crackles in both lungs. Examination was otherwise normal with no signs of connective tissue disease.

A chest radiograph showed bilateral patchy alveolar opacities with prominent involvement of both lower lungs (Fig. 1A). High-resolution computed tomography (HRCT) demonstrated multiple areas of subpleural and peribronchial patchy consolidation with ground glass opacities (Fig. 1B).

Laboratory tests showed a white blood cell count of $10,400 / \mathrm{mm}^{3}$, a hemoglobin concentration of $13.3 \mathrm{~g} / \mathrm{dL}$, a platelet count of $328,000 / \mathrm{mm}^{3}$, an erythrocyte sedimentation rate of $47 \mathrm{~mm} / \mathrm{hr}$, and C-reactive protein levels of $12.2 \mathrm{mg}$ / dL. Oxygen saturation was $89 \%$ in room air. Serum chemistry and immunologic data, including autoantibodies for col-

${ }^{1}$ Department of Internal Medicine, School of Medicine, Kyungpook National University, Korea and ${ }^{2}$ Department of Pathology, School of Medicine, Kyungpook National University, Korea

Received for publication July 19, 2010; Accepted for publication November 1, 2010

Correspondence to Dr. Chang Ho Kim, kimch@knu.ac.kr 

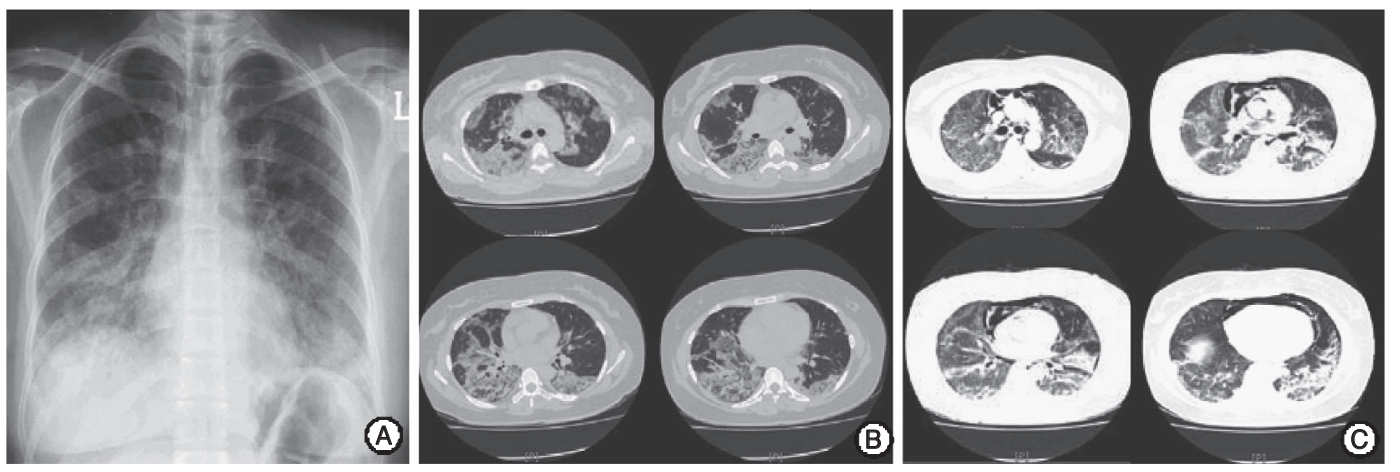

Figure 1. (A) The initial chest radiograph showed patchy alveolar opacities predominantly in the lower lobes. (B) High-resolution computed tomography images demonstrated patchy and randomly distributed consolidation and ground glass attenuation throughout both lungs. $(C)$ Fifteen days after admission, computed tomography scan revealed pneumomediastinum and pneumothorax.

Table 1. Results of Chemistry and Immunology Tests

\begin{tabular}{|c|c|c|}
\hline Test & Result & Reference range \\
\hline \multicolumn{3}{|l|}{ Chem istry } \\
\hline Blood urea nitrogen & 11.4 & $6-20 \mathrm{mg} / \mathrm{dL}$ \\
\hline Creatinine & 0.57 & $0.6-1.3 \mathrm{mg} / \mathrm{dL}$ \\
\hline Aspartate aminotransferase & 38 & $0-37 \mathrm{U} / \mathrm{L}$ \\
\hline Alanine am inotransferase & 20 & $0-41 \mathrm{U} / \mathrm{L}$ \\
\hline Ald olase & 17.9 & $0-7.6 \mathrm{U} / \mathrm{L}$ \\
\hline Creatine phosphokinase & 42 & $\leq 170 \mathrm{IU} / \mathrm{L}$ \\
\hline \multicolumn{3}{|l|}{ Im munology } \\
\hline AN A & Neg ative & Neg ative \\
\hline Anti-d sDN A & 0.1 & $0-7 \mathrm{IU} / \mathrm{mL}$ \\
\hline $\mathrm{RF}$ & 10 & $0-14 \mathrm{IU} / \mathrm{mL}$ \\
\hline Anti-CCP antibody & 9.55 & $0-17 \mathrm{U} / \mathrm{mL}$ \\
\hline Anti-SSA/Ro antibody & Neg ative (32.59) & $\mathrm{Neg}$ ative $(<150 \mathrm{AAU} / \mathrm{mL})$ \\
\hline Anti-SSB/La antibody & Neg ative $(40.24)$ & Neg ative $(<150 \mathrm{AAU} / \mathrm{m} \mathrm{L})$ \\
\hline Anti-Scl-70 antibody & Neg ative & Neg ative \\
\hline Anticentromere antibody & Neg ative & Neg ative \\
\hline Anti-Jo-1 antibody & Neg ative & Neg ative \\
\hline Anti-RNP antibo dy & 9.53 & $0-25 \mathrm{U} / \mathrm{mL}$ \\
\hline AN CA & Neg ative & Neg ative \\
\hline
\end{tabular}

AN A : antinuclear antibody; dsDN A: double-stranded DNA; RF: rheum atoid factor; CCP: cyclic citrullinated peptide; SS: SjOgren's syndrom e; Scl: scleroderma; Jo-1: histidyl transfer RNA synthetase; RNP: rib onucleoprotein; ANCA: antineutrophil cytoplasmic antibody.

lagen vascular diseases, were unremarkable (Table 1). Serologic tests for atypical organisms and human immunodeficiency virus were negative. A nasopharyngeal swab test for potentially pathogenic viruses was also negative.

Cellular analysis of the bronchoalveolar lavage fluid revealed increased neutrophils (26\%) and lymphocytes (17\%) and a decreased $\mathrm{CD} 4^{+} / \mathrm{CD}^{+}$ratio of 0.66 . No pathogen was cultured. A transbronchial lung biopsy specimen exhibited chronic inflammatory changes. Empirical broad antibiotics were initially administered. Over the subsequent 3 days, the patient's condition continued to deteriorate with worsening hypoxemia and chest radiograph findings. Prednisolone was administered (60 $\mathrm{mg}$ total, $1 \mathrm{mg} / \mathrm{kg} /$ day) in addition to the antibiotics, as there was a possibility that the patient had OP based on the clinico-radiologic findings. Nevertheless, the patient was transferred to the intensive care unit two days later due to intractable hypoxemia. Methylprednisolone treatment was then commenced at a rate of $125 \mathrm{mg}$ injected every 6 hours for 3 consecutive days, but despite this, arterial blood gas under oxygen inhalation of $15 \mathrm{~L} / \mathrm{min}$ was $\mathrm{pH}$ 7.53, $\mathrm{PaCO}_{2} 32 \mathrm{mmHg}$, and $\mathrm{PaO}_{2} 65 \mathrm{mmHg}$. As shown in Fig. 2, oral therapy with $150 \mathrm{mg}(2.5 \mathrm{mg} / \mathrm{kg}) \mathrm{CsA}$ and macrolide was initiated and other antibiotics were discontinued. Following commencement of this regimen, chest radiograph findings and hypoxemia stopped deteriorating and the patient's dyspnea began to improve. Though several signs of improvement were noted, on day 5 after administration of CsA and macrolide the patient complained of lower left chest pain. A follow-up chest radiograph and CT scan revealed pneumomediastinum and pneumothorax (Fig. 1C). A thoracoscopic lung biopsy was performed to obtain an accurate pathologic diagnosis as well as to manage the air leak problem. The biopsy showed histopathologic changes characteristic of OP in that the alveoli and alveolar ducts were 

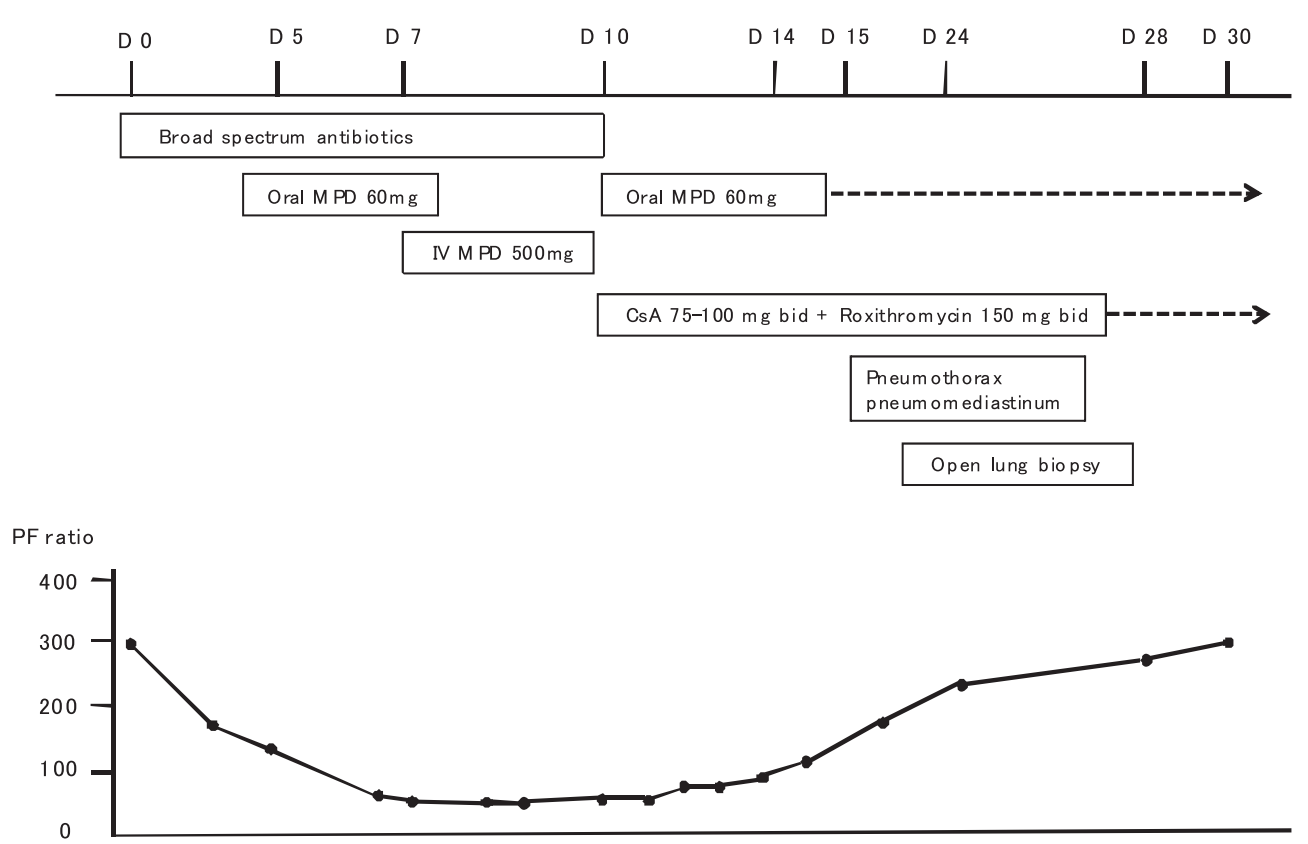

Figure 2. The clinical course of the patient. D: admission day, MPD: methylprednisolone, CsA: cyclosporin A, PF ratio: $\mathrm{PaO}_{2} / \mathrm{FiO}_{2}$
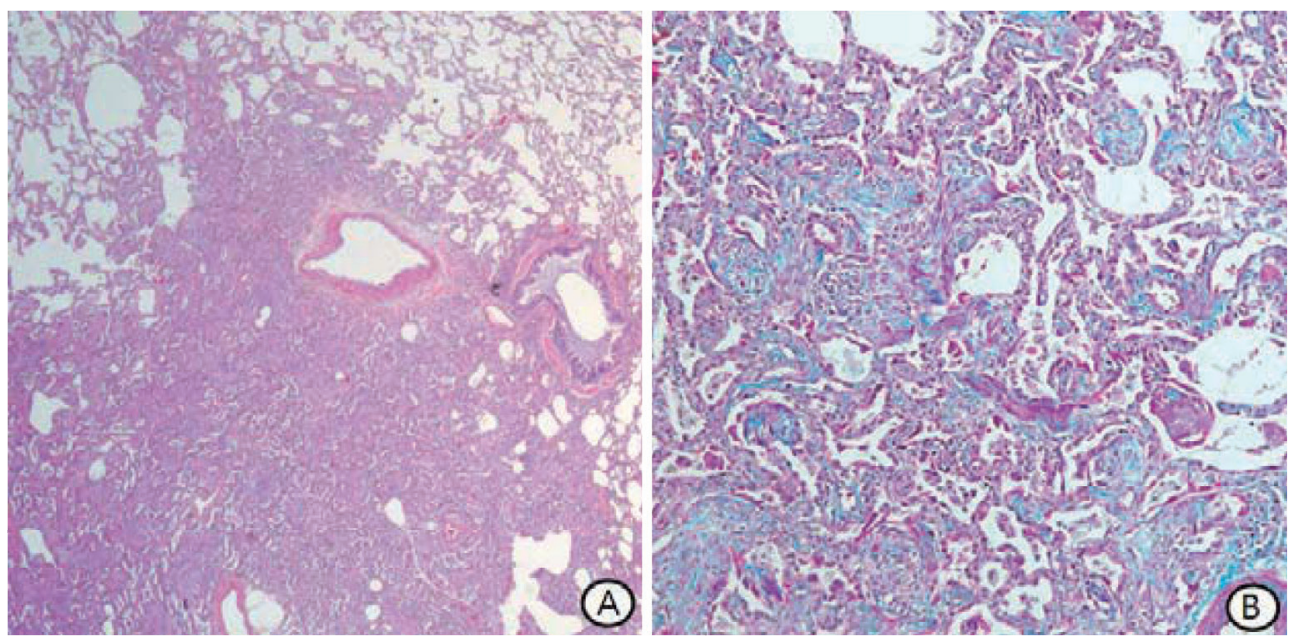

Figure 3. Microscopic findings. (A) Patchy fibrosis was observed and was sharply demarcated from adjacent areas of normal parenchyma (Hematoxylin and Eosin staining $\times 20$ ). (B) The typical fibroblast plugs occluded airspaces and some fibroblast plugs were noted in the interstitium (Trichrome $\times 100$ ).

filled with buds of granulation tissue (Fig. 3A, 3B).

The chest tube was safely removed after 9 days, and the patient was moved out of intensive care with gradual improvement in arterial oxygenation. Chest radiograph findings and the arterial oxygenation index improved steadily, resulting in oxygen saturation of $92 \%$ in room air 4 weeks after administration of CsA and macrolide together with corticosteroids. Pulmonary function tests were performed 5 weeks after admission and showed a FVC of $52 \%$ of the predicted value and $\mathrm{FEV}_{1}$ of $62 \%$ of the predicted value, yielding a $\mathrm{FEV}_{1} / \mathrm{FVC}$ of $92 \%$, and a diffusing capacity of $31 \%$ of the predicted value. At discharge, $125 \mathrm{mg} /$ day of $\mathrm{CsA}$ and macrolide in addition to $50 \mathrm{mg}$ /day prednisolone were prescribed. Prednisolone and CsA treatment was slowly tapered off over time in outpatient follow-up visits. Six months later, CsA treatment was halted while $7.5 \mathrm{mg} /$ day prednisolone was continued, and no relapse occurred. A follow-up chest radiograph and HRCT at 6 months postdischarge showed that the lungs were largely clear but had slight fibrotic changes in the peripheral lower region (Fig. 4A, 4B). At 6 months, lung function has also improved with a FVC and a $\mathrm{FEV}_{1}$ of $85 \%$ and $99 \%$ of the predicted value, respectively, and a $\mathrm{DL}_{\mathrm{co}}$ of $69 \%$ of the predicted value. 

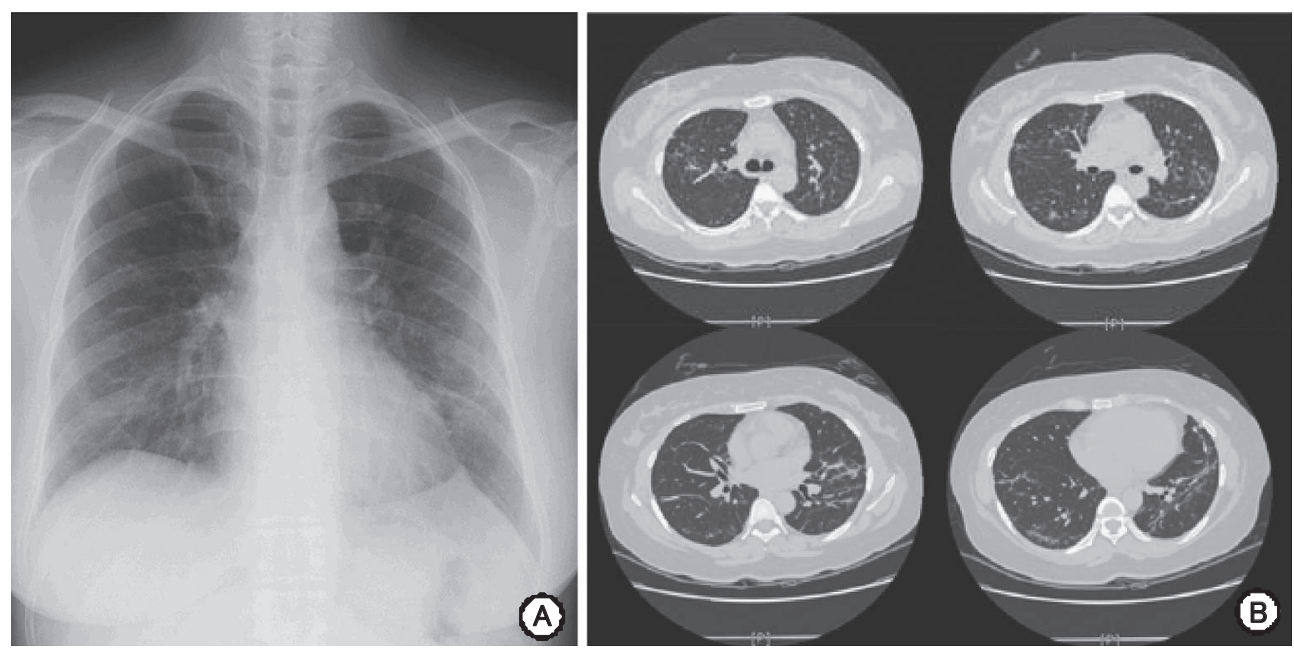

Figure 4. Chest radiograph findings six months post treatment. (A) A chest radiograph showed resolution of the previously observed bilateral patchy consolidations in both lungs. (B) High-resolution computed tomography images revealed marked improvement in the consolidation as well as some fibrotic changes in the peripheral region of the lung.

\section{Discussion}

OP generally responds well to steroids and has an excellent prognosis. However, in 10 to $15 \%$ of OP patients, the disease is steroid-resistant and progressive $(6,8)$. In rapidly progressive cases that show increased neutrophil counts in the BAL fluid, as in the present patient, OP must be differentiated from acute interstitial pneumonitis, acute fibrous organizing pneumonia, and other similar diseases. We thus performed an open lung biopsy, which excluded conditions other than OP. The factors associated with a poor prognosis in OP cases remain unclear, although some have noted that OP patients with autoimmune etiology tend to do worse (1). Reasonable efforts were made to rule out subclinical connective tissue disease with a high index suspicion, but all of the examined autoantibody tests were within the normal range. The present patient was presumed to have COP although we cannot completely exclude the possibility of occult connective tissue disease.

Corticosteroids are the conventional initial treatment for OP. In general, clinical improvement is seen within several days $(1,3,4,11)$, and the response is quite dramatic in some patients. Accordingly, we administered prednisolone at an adequate dose for a duration, which has resulted in the clear improvement in most cases. However, the patient's condition worsened and was thus presumed to be a rapidly progressive COP case without prompt response to steroid.

Minimal data have been reported regarding alternative immunosuppressive agents in cases of corticosteroid refractory OP (9-13). Some reports suggest that early treatment with cyclophosphamide can be effective, especially in patients that fail to respond to treatment with corticosteroids alone $(9,11)$. In contrast, Chang et al (13) described two rapidly progressive OP patients that ultimately died after having received $1,000 \mathrm{mg} /$ day cyclophosphamide intravenously in addition to corticosteroid therapy. Yet, administration of CsA together with a corticosteroid in a fulminant OP patient resulted in a successful outcome (10). In that case, CsA was administered for 4 months without serious side effects and the authors conclude that further study is warranted to define the utility of CsA therapy for corticosteroidresistant OP. Two radiologically suspected OP cases associated with juvenile dermatomyositis have also been described in which early commencement of CsA was beneficial (12). Since cyclophosphamide treatment led to the conflicting results and favorable outcomes were achieved with CsA, we chose to use CsA as a non-steroidal treatment for our patient.

CsA is an immunomodulatory drug that plays a role in inhibiting the activation of helper $\mathrm{T}$ cells and alveolar macrophages (14), and has been used to treat several types of pulmonary interstitial diseases (15-17). The pathogenesis of OP remains speculative. One hypothesis is that exposure to inhaled antigens stimulates inflammation and lymphokine production in bronchus-associated lymphoid tissue (3), initiating OP-associated immune responses. CsA could help suppress these inflammatory and immune processes.

We also added macrolide to our treatment regimen. Macrolides may be adjuvant therapy in OP patients receiving steroids for a prolonged periods or as an alternative to corticosteroids treatment $(18,19)$. These drugs are thought to act mainly as immunomodulatory agents rather than targeting infectious organisms directly. We thus treated the present patient with macrolide in combination with CsA and corticosteroid. However, further testing in OP is required before definitive recommendations can be made.

Pneumothorax and pneumomediastinum presentation in OP patients is rare $(20,21)$. Kofteridis et al $(21)$ reported a fatal case of OP in which a spontaneous pneumothorax de- 
veloped and the patient failed to survive despite corticosteroid treatment. In this study, the authors note that spontaneous pneumothorax is a rare complication of OP and may adversely affect prognosis. Another case of OP has been documented that presented as a pneumothorax, pneumomediastinum, and subcutaneous emphysema, and a good outcome was obtained with corticosteroid in this patient (20). In this report, we document the successful management of a patient with COP complicated by air leak syndrome. The prognostic impact of air leak syndrome as a complication in COP seems to be rather uncertain.

We obtained a good outcome with combined CsA and macrolide as adjunctive drugs in rapidly progressive COP without prompt steroid-response. However, we cannot exclude the possibility of a delayed corticosteroid effect regardless of an administration of CsA and macrolide. Furthermore, CsA and macrolide were simultaneously initiated. Thus, we cannot discriminate which drug ultimately contributed to disease resolution. However, it is difficult to only wait for a delayed effect of corticosteroid under worsening condition of the patient and perform controlled studies in COP patients to determine the effects of alternative treatment regimens due to the rarity of $\mathrm{COP}$ with steroidresistance or delayed steroid-response. In addition, delaying treatment in rapidly progressive patients receiving steroid therapy could lead to poor outcomes. It may be important to begin aggressive therapy before irreversible lung changes develop. Additional reports regarding non-steroid agent treatment in such cases are needed to improve treatment recommendations.

Our findings suggest that $\mathrm{CsA}$ and macrolides may be beneficial in rapidly progressive COP patients without a timely response to steroid treatment, and thus could be considered early for therapeutic trials in these cases.

The authors state that they have no Conflict of Interest (COI).

\section{References}

1. Epler GR, Colby TV, McLoud TC, Carrington CB, Gaensler EA. Bronchiolitis obliterans organizing pneumonia. N Engl J Med 312: 152-158, 1985.

2. American Thoracic Society; European Respiratory Society. American Thoracic Society/European Respiratory Society International Multidisciplinary Consensus Classification of the Idiopathic Interstitial Pneumonias. This joint statement of the American Thoracic Society (ATS), and the European Respiratory Society (ERS) was adopted by the ATS board of directors, June 2001 and by the ERS Executive Committee, June 2001. Am J Respir Crit Care Med 165: 277-304, 2002.

3. Schlesinger C, Koss MN. The organizing pneumonias: an update and review. Curr Opin Pulm Med 11: 422-430, 2005.

4. Drakopanagiotakis F, Polychronopoulos V, Judson MA. Organizing pneumonia. Am J Med Sci 335: 34-39, 2008.

5. Iannuzzi MC, Farhi DC, Bostrom PD, Petty TL, Fisher JH. Fulminant respiratory failure and death in a patient with idiopathic bronchiolitis obliterans. Arch Intern Med 14S: 733-734, 1985.

6. Cohen AJ, King TE Jr, Downey GP. Rapidly progressive bronchiolitis obliterans with organizing pneumonia. Am J Respir Crit Care Med 149: 1670-1675, 1994.

7. Nizami IY, Kissner DG, Visscher DW, Dubaybo BA. Idiopathic bronchiolitis obliterans with organizing pneumonia: an acute and life-threatening syndrome. Chest 108: 271-277, 1995.

8. Yousem SA, Lohr RH, Colby TV. Idiopathic bronchiolitis obliterans organizing pneumonia/cryptogenic organizing pneumonia with unfavorable outcome: pathologic predictors. Mod Pathol 10: 864871, 1997.

9. Purcell IF, Bourke SJ, Marshall SM. Cyclophosphamide in severe steroid-resistant bronchiolitis obliterans organizing pneumonia. Respir Med 91: 175-177, 1997.

10. Koinuma D, Miki M, Ebina M, et al. Successful treatment of a case with rapidly progressive bronchiolitis obliterans organizing pneumonia (BOOP) using cyclosporin A and corticosteroid. Intern Med 41: 26-29, 2002.

11. King TE Jr, Mortenson RL. Cryptogenic organizing pneumonitis. Chest 102: 8S-13S, 1992.

12. Kobayashi I, Yamada M, Takahashi Y, et al. Interstitial lung disease associated with juvenile dermatomyositis: clinical features and efficacy of cyclosporin A. Rheumatology 42: 371-374, 2003.

13. Chang J, Han J, Kim D, et al. Bronchiolitis obliterans organizing pneumonia: clinicopathologic review of a series of 45 Korean patients including rapidly progressive form. J Korean Med Sci 17: 179-186, 2002.

14. Losa García JE, Mateos Rodríguez F, Jiménez López A. Effect of cyclosporin A on inflammatory cytokine production by human alveolar macrophages. Respir Med 92: 722-728, 1998.

15. Inase N, Sawada M, Ohtani Y, et al. Cyclosporin A followed by the treatment of acute exacerbation of idiopathic pulmonary fibrosis with corticosteroid. Intern Med 42: 565-570, 2002.

16. Homma S, Sakamoto S, Kawabata M, et al. Cyclosporin treatment in steroid-resistant and acutely exacerbated interstitial pneumonia. Intern Med 44: 1144-1150, 2005.

17. Sakamoto S, Homma S, Miyamoto A, Kurosaki A, Fujii T, Yoshimura K. Cyclosporin A in the treatment of acute exacerbation of idiopathic pulmonary fibrosis. Intern Med 49: 109-115, 2010.

18. Ichikawa Y, Ninomiya H, Katsuki M, Hotta M, Tanaka M, Oizumi $\mathrm{K}$. Low-dose/long-term erythromycin for treatment of bronchiolitis obliterans organizing pneumonia (BOOP). Kurume Med J 40: 6567, 1993.

19. Stover DE, Mangino D. Macrolides: a treatment alternative for bronchiolitis obliterans organizing pneumonia? Chest 128: 36113617, 2005.

20. Iwanaga $T$, Hirota $T$, Ikeda $T$. Air leak syndrome as one of the manifestations of bronchiolitis obliterans organizing pneumonia. Intern Med 39: 163-165, 2000.

21. Kofteridis DP, Bouros DE, Vamvakas LN, et al. Pneumothorax complicating fatal bronchiolitis obliterans organizing pneumonia. Respiration 66: 266-268, 1999.

(C) 2011 The Japanese Society of Internal Medicine http://www.naika.or.jp/imindex.html 